\title{
De-construcción de las unidades formadoras de profesores en Colombia
}

\section{De-construction of teacher-training units in Colombia}

\author{
Nelson Ernesto López Jiménez \\ Grupo de Investigación PACA \\ nelopez53@gmail.com \\ María Fernanda Pérez Gutiérrez \\ Grupo de Investigación PACA \\ nanita_12_88@hotmail.com \\ Wilson Roberto Perdomo Cortes \\ Grupo de Investigación PACA \\ wilsonperdomo07@gmail.com
}

\begin{abstract}
No habremos engendrado ciudadanos verdaderamente libres en el sentido socrático a menos que formemos personas capaces de razonar por sí mismas y argumentar correctamente, capaces de entender la diferencia entre un razonamiento con validez lógica y otro lógicamente débil, de distinguir entre la estructura lógica de un discurso y la verdad de sus premisas.

(Martha C. Nussbaum El cultivo de la humanidad, 2005)
\end{abstract}

\section{Resumen}

El presente artículo da cuenta del desarrollo y resultados de la investigación “Evaluación de los Programas de Licenciatura de la Facultad de Educación de la Universidad Surcolombiana de Neiva", que pretende re-significar los procesos desarrollados por las IES para la obtención de los Registros Calificados ${ }^{1}$, como una oportunidad para propiciar la discusión y reflexión colectiva entorno al dilema existente que surge en los procesos de evaluación de los Programas Académicos. El objetivo central de la investigación fue realizar un proceso de evaluación endógeno de los Programas de Licenciatura que ofrece la Facultad de Educación de la Universidad Surcolombiana que aluda las dimensiones administrativa, académica e investigativa en las cuales se desarrollan.

Como conclusión general, los procesos de autoevaluación de los Programas Académicos, con miras a la obtención del Registro Calificado y/o la Acreditación de Calidad, es una oportunidad de introspección real, honesta y rigurosa que permite construir Planes de Acción, coherentes con las problemáticas internas y externas que permite fortalecer y consolidar la calidad académica de los programas.

Si bien el Ministerio de Educación Nacional y Consejo Nacional de Acreditación -CNA- tienen unos parámetros establecidos para evaluar procesos académicos y administrativos, se observa que son requerimientos técnicos, que se asumen como requisitos y exigencias en los Programas, alejadas de la realidad institucional que no garantizan la pertenencia social y la pertinencia académica de los Programas.

1 Proceso regulado por el Decreto 1295 de 2010 integrado al Decreto Compilatorio 1075 de mayo de 2015. 
Se halló una tendencia generalizada en afirmar que aunque se hacen Planes de Acción y de Mejoramiento en los Programas Académicos de las Universidades Públicas para alcanzar niveles de calidad educativa, existe desinterés de parte de las instituciones encargadas de diseñar las políticas públicas, carencia de financiamiento por parte de las organizaciones gubernamentales y poca gestión institucional para desarrollar las estrategias que permitan transformar la educación de cara a las necesidades de la sociedad.

Palabras clave: Evaluación, Programas académicos, autoevaluación, Registro Calificado y Acreditación de Calidad.

\section{Introducción}

La problemática de las unidades formadoras de docentes en Colombia se convierte en un tema recurrente cuando se aborda el análisis relacionado con el papel que juega la Educación como proceso esencial en el desarrollo de un país, como también, la responsabilidad que le atañe a los profesionales "destinados históricamente" para ejercer y desempeñarse como maestros, en la construcción de identidad, de ciudadanía, de cultura, de valores, de principios, de construcción de futuro, de sentido de paz, equidad y reconciliación.

En desarrollo de lo regulado en las políticas públicas que rigen la Educación Superior en Colombia, las Instituciones de Educación Superior -IES- deben cumplir con una serie de exigencias y requisitos que garanticen las condiciones básicas de calidad y rigor formativo, lo cual les permitirá la obtención del Registro Calificado (entendido como la licencia de funcionamiento de los programas) y la Acreditación Institucional y de Programas (reconocimiento del cumplimiento de condiciones de excelencia).

En este contexto, las Facultades de Educación, y concretamente, la de la Universidad Surcolombiana, desarrolla procesos de autoevaluación y heteroevaluación como garantes de seriedad, rigor y solidez del proceso de formación de profesionales en el campo de la Educación (licenciados).

2 Consejo Nacional de Ciencia y Tecnología de México.
La presente investigación tiene como finalidad realizar un proceso de indagación, reflexión y análisis de las acciones evaluativas desarrolladas por los programas de pregrado de la Facultad de Educación en la USCO, en el marco del proceso de obtención de los Registros Calificados y algunas Acreditaciones de Alta Calidad.

\section{Estado del arte}

Teniendo en cuenta que la presente investigación analizó el actual portafolio académico de los programas de pregrado de la Facultad de Educación de la Universidad Surcolombiana, es pertinente referenciar algunos estudios relacionados con la investigación evaluativa de los programas de pregrado a nivel internacional y a nivel nacional que permitió el fortalecimiento del trabajo investigativo.

En el año 2009, el Instituto Politécnico Nacional Escuela Superior de Comercio y Administración en México realizó la investigación "Evaluación de la Organización Académico-Administrativa de tres Programas de Maestría en Educación en relación con los parámetros establecidos por el CONACYT"2. El objetivo de esta investigación, fue "evaluar la organización académico y administrativa de tres programas de Maestría en Educación, en relación con los parámetros del Programa Integral de Fortalecimiento al Posgrado (PIFOP) establecido por el Consejo Nacional de Ciencia y Tecnología (CONACYT)". 
Los instrumentos utilizados en el trabajo de campo fueron, la entrevista dirigida a cada uno de los coordinadores de los tres programas de Maestría en Educación, el cuestionario orientado a los docentes de la planta académica de cada programa de Maestría y el inventario que se realizó a partir de una guía, cuyo objetivo principal fue recolectar los datos sobre las características que posee la infraestructura de apoyo y de equipamiento como los Centros de Documentación de cada uno de los programas de Maestría.

En el desarrollo de la investigación, los autores Oliver Cardoso Édgar, Trinidad Cerecedo María y Trejo Cázares Carmen, precisan que la evaluación de un programa educativo es un proceso orientado a identificar las fortalezas y las debilidades de los programas.

La Escuela Superior de Comercio y Administración (ESCA) Instituto Politécnico Nacional de México en el 2011, desarrolló la investigación "Evaluación de la Organización Académico Administrativa con Base en la Metodología de los Sistemas Suaves: El Caso de la Licenciatura en Turismo", cuyo objetivo fue "evaluar la organización académico administrativa de tres programas de licenciatura en Turismo, con el propósito de identificar las características según los criterios del Consejo para la Acreditación de la Educación Turística (CONAET) y TedQual (Tourism Education Quality)"; su metodología se caracterizó por ser evaluativa y descriptiva de tipo transversal, la cual permitió la observación de las situaciones y los hechos generados en el contexto.

Para la realización de esta investigación se utilizaron como instrumentos el cuestionario y la guía de observación, con el fin de recolectar la información necesaria para analizarla cualitativamente (mediante el Atlas Ti) y cuantitativamente (a través de la estadística descriptiva). En los resultados obtenidos de la investigación, el programa de Licenciatura en Turismo, ofrecida por la Escuela Superior de Turismo (EST), de México, cumplió con los criterios de certificación. Se consideró que sus instalaciones y el programa ofertado se encontraron de acuerdo con los lineamientos de certificación.

La Universidad de Valencia en España realizó una investigación de corte cuantitativo denominado "Patrones Metodológicos en la Investigación Española sobre Evaluación de Programas Educativos (2004)", su metodología se orientó en la descripción de los factores y variables existentes de los programas académicos.

Esta investigación destacó en sus conclusiones que las investigaciones sobre evaluación de programas se sustentan en el análisis de datos numéricos. Es preciso mencionar que la evaluación de programas suele realizarse en contextos sociales de enorme complejidad, por ello abundan realizaciones en cuyo diseño no siempre es posible el control total de todas las variables, como los de tipo cuasi-experimental o descriptivos (Tejedor, 2000).

Los estudios relacionados con la investigación evaluativa de los programas universitarios a nivel internacional, pretenden aportar una serie de acciones y estrategias que garantizan el funcionamiento en los programas, teniendo en cuenta los aspectos académicos y administrativos exigidos por las entidades encargadas de evaluar los programas. Los resultados de dichos estudios se sustentan en la recolección de datos y análisis de los mismos, con el propósito de mejorar las acciones académicas y administrativas de los programas según los parámetros de calidad.

De otra parte, es necesario precisar algunas investigaciones a nivel nacional para analizar las diferencias o semejanzas con las investigaciones evaluativas de los programas a nivel internacional.

En Colombia se realizó un estudio en el año 2006 sobre "Evaluación de los Sistemas de Gestión de Calidad en los Programas de Ingeniería Industrial de Barranquilla". (Universidad Autónoma del Caribe, Universidad del Atlántico, Corporación Universitaria de la Costa, Universidad Libre, Politécnico de la Costa Atlántica, Universidad Simón Bolívar y Universidad Antonio Nariño), posteriormente se realizó un estudio de 
actualización en el año 2009, de los diferentes Programas de Ingeniería Industrial.

La naturaleza de la investigación fue de tipo descriptivo, cualitativo y exploratorio, para la evaluación de los programas se realizaron entrevistas que permitieron el análisis de la información teniendo en cuenta los actores, las relaciones básicas y la situación general de contexto. Esta investigación se orientó a la caracterización de los Sistemas de Gestión de la Calidad de los diferentes Programas de Ingeniería Industrial, como la conceptualización del Sistema de Gestión de la Calidad ISO 9001:2008.

De acuerdo con los resultados obtenidos, los Programas de Ingeniería Industrial, no tienen un Sistema de Gestión de la Calidad que responda a las exigencias de los estándares de calidad ISO 9001: 2008, por tal razón, los programas no trabajan de manera articulada las acciones de cada organización ya que no poseen estructuras de organización para planificar, vigilar y mejorar el Sistema de Gestión de la Calidad. Esta investigación intenta convertirse en una herramienta metodológica y practica en el mejoramiento de dichos programas.

En la Universidad del Valle se publicó a principios del año 2008 una investigación denominada "Evaluación del desempeño docente: la experiencia del Programa Académico de Odontología de la Universidad del Valle, 2005-2007 Colombia Médica"; cuyo propósito principal fue "analizar los resultados del desempeño docente según una encuesta realizada a los estudiantes del Programa Académico de Odontología entre el año 2005 y el año 2007", que determinó la diferencia significativa en el desempeño docente según la categoría y las áreas de formación del programa. Este estudio fue de carácter descriptivo de corte transversal.

En la investigación, se señala que la evaluación docente es una reflexión permanente sobre la práctica que permite aportar al proceso elementos fundamentales para el mejoramiento. El sistema de evaluación brinda información relevante y pertinente para el diseño de estrategias de intervención tanto en el diseño curricular como en las acciones de la formación docente.

En el año 2008 la Universidad del Norte, en Barranquilla realizó un estudio descriptivo denominado "Factores Asociados al fracaso académico en estudiantes universitarios de Barranquilla (Colombia) Psicología desde el Caribe".

Una de las audiencias que participaron en este estudio fueron los estudiantes que asistían a un programa exclusivo, con bajo rendimiento académico y riesgo de salir del programa. Específicamente se utilizó como instrumento una entrevista semiestructurada previamente elaborada para el Programa de Apoyo y Seguimiento, los aspectos evaluados fueron los siguientes: datos de identificación personal; antecedentes académicos; antecedentes familiares; características personales; situación académica actual. De acuerdo con los aspectos señalados, se evidenció que la mayoría de los estudiantes no reciben una orientación vocacional adecuada antes de ingresar a los estudios superiores. Además, presentan limitaciones en las competencias de concentración y atención, en la distribución del tiempo, en la deficiente utilización de técnicas de estudio, y en la poca asistencia a clases por la baja motivación en las actividades académicas y la relación con la familia.

No obstante, este estudio señala que las acciones para reducir el fracaso deben ser emprendidas antes del ingreso a la universidad, con estrategias de estudio y afrontamiento que les brinden a los jóvenes las mejores opciones entre sus intereses y capacidades con las oportunidades que ofrece la sociedad.

En los hallazgos encontrados de este estudio, se menciona la necesidad de implementar una investigación evaluativa que analice de manera integral los procesos internos de los programas académicos.

El Consejo Nacional de Acreditación CNA en Colombia determina las exigencias que deben cumplir las IES que pretenden acreditarse de Alta Calidad. En estos requerimientos se contemplan las exigencias institucionales y de programas 
académicos para reconocer el cumplimiento de los niveles de excelencia. La acreditación de alta calidad es un proceso voluntario que se inicia con acciones de Autoevaluación que desarrollan las IES y a partir de sus resultados, se solicita al CNA se programe la visita de pares académicos para verificar las condiciones iniciales, y de encontrar el cumplimiento de los criterios y exigencias se autoriza el desarrollo pleno del proceso de Acreditación de Calidad, sea este institucional o de programas. La Acreditación de Alta Calidad está regulada por la Ley 30 de 1992 mediante la cual se organiza el servicio público de la Educación Superior.

La CONACES, Comisión Intersectorial para el mejoramiento de la calidad de la Educación Superior, forma parte del SACES, Sistema de Aseguramiento de la Calidad de la Educación Superior, tienen como función esencial verificar el cumplimiento de los requisitos de calidad de los programas académicos que ofrecen las IES en sus diferentes niveles (Técnico Profesional, Tecnológico, Profesional o Universitario y de Postgrado, Especialización, Maestría y Doctorado) que solicitan el Registro Calificado para poder ofrecer sus programas académicos. El Registro Calificado es una exigencia de ley, es decir, de obligatorio cumplimiento, si un programa no cuenta con Registro Calificado, no puede ofrecerse.

Es preciso señalar que los programas que ofrece la Facultad de Educación de la Universidad Surcolombiana, tres cuentan con Acreditación de Calidad y TODOS cuentan con el Registro Calificado. No obstante, una de las razones de esta investigación es insistir en una evaluación endógena, interna que avance en una investigación evaluativa de los programas de pregrado que analice las dimensiones administrativa, académica e investigativa de los procesos que se desarrollan en el interior de los programas. Es necesario insistir que la autonomía universitaria permite que los procesos se desarrollen, no necesariamente, hacia el cumplimiento de requisitos, sino con el propósito de consolidar su proyecto formativo y eso es lo que persigue esta investigación. Se trata de "ampliar" el horizonte de realización de la evaluación y autoevaluación de programas de la Facultad de Educación.

Cuando se hace referencia a la evaluación, ya sea del rendimiento académico de los estudiantes o de los programas e instituciones, hay que advertir la naturaleza polisémica de este concepto, es decir, es preciso señalar que la evaluación tiene más de una interpretación y concepción, de ella se desprenden diversos posiciones a partir de los contextos, las necesidades a abordar, y los objetivos que se pretenden alcanzar. La evaluación tiende a asociarse a significados como; verificar, interpretar, medir, estimar, comprender, valorar, juzgar, apreciar y rendir que permiten configurar un campo $^{3}$ conformado por las diferentes posiciones, argumentos e interpretaciones existentes sobre la evaluación, que no se expresan de manera estática, sino que por el contrario, avanzan en la consecución de "dominio" sobre el campo, lo cual permite, la hegemonía o imposición de sus planteamientos, prácticas y desarrollos. Por definición todo proceso de evaluación busca generar alguna modificación en la realidad que es evaluada. Se evalúa un aspecto de la realidad educativa (un sistema, un programa, los logros de los estudiantes, el desempeño de un docente) con el propósito, primero de comprender mejor y valorar dicha realidad y, segundo, de contribuir a cambiarla para mejorarla"4

\section{Metodología}

Teniendo en cuenta la naturaleza de la problemática a abordar y el interés que existe

3 La noción de campo es entendida "como un estado de relaciones de fuerza entre los agentes o las instituciones envueltas en la lucha por la distribución del capital específico que acumulado en el curso de las luchas anteriores orienta las estrategias posteriores. Las luchas en el campo tienen por efecto el monopolio de la vigencia legítima (autoridad específica), es decir, la conservación o la subversión de la estructura de la distribución del capital específico" (Bourdieu, 1995).

4 La evaluación externa en seis países de América Latina: Balances y retos, Ed. Bonaventuriana. 
de propiciar espacios y escenarios para que los protagonistas de la acción evaluativa de los programas académicos expresen sus puntos de vista en relación con la realidad actual de la formación del profesional de la educación, el carácter de esta investigación es cualitativo; persigue crear las condiciones para que la "voz de los actores" encuentre un escenario propicio para establecer diálogos intencionados en torno a lo que realmente acontece en el proceso de formación de profesionales de la Educación. Se insiste en enfatizar las miradas y percepciones que los actores del proceso (directivos, docentes, estudiantes) tienen de cara a la realidad que están viviendo.

Se intenta describir y analizar la realidad institucional de cada Programa en comparación con la situación legal (Acreditación de Alta Calidad y Registro Calificado) ante el Ministerio de Educación Nacional. Se pretendió realizar un diagnóstico detallado de cada Programa que desarrolló un proceso de auto-reconocimiento de los problemas, fortalezas y necesidades institucionales. A través de la inmersión en la situación real de los Programas, se estructuró una propuesta alternativa en el campo académico, administrativo e investigativo de los Programas de pregrado de la Facultad de Educación de la Universidad Surcolombiana.

De esta realidad se decidió realizar una Entrevista a profundidad a los siguientes estamentos:

Los Directivos: Jefes de Programa

Profesores de los programas: docentes tiempo completo y de cátedra Estudiantes: a partir del sexto semestre (se indagó sobre los procesos de autoevaluación de los programas que se consideraron básicos para la obtención del Registro Calificado, el Equipo Investigador tomó la decisión de consultar a estudiantes que hayan desarrollado las 3 cuartas partes de su programa académico).

\section{Análisis y conclusiones}

El Grupo de Investigación PACA, Categoría A de Colciencias, considera que la autoevaluación de los Programas Académicos, con miras a la obtención del Registro Calificado y/o la Acreditación de Calidad, es una oportunidad de introspección real, honesta y rigurosa que permite construir Planes de Acción, coherentes con las problemáticas internas y externas para fortalecer y consolidar la calidad académica de los programas.

Se entiende por calidad académica de los Programas la coherencia sostenible y básica entre los planteamientos teóricos (Propósitos de Formación) y sus desarrollos prácticos de cara a las problemáticas y necesidades de la sociedad que pretende intervenir.

En esa medida, evaluar los Programas de Pregrado de la Facultad de Educación implica determinar su pertenencia social y pertinencia académica y entender la importancia del proceso de formación del profesional de la educación en Colombia.

En cuanto, al Registro Calificado y la Acreditación de Alta Calidad de las Licenciaturas de la Facultad de Educación, los docentes y Jefes de Programa entrevistados consideran que su obtención no garantiza que los Programas Académicos sean rigurosos, excelentes y sólidos, pues determinan que los procesos administrativos son débiles, engorrosos, burocráticos e ineficientes y que la Universidad no cuenta con infraestructura, recursos y docentes suficientes para considerase como Programas de alta calidad educativa.

Si bien el Ministerio de Educación Nacional y Consejo Nacional de Acreditación -CNA- tienen unos parámetros establecidos para evaluar procesos académicos y administrativos, se observa que son requerimientos técnicos, que se asumen como requisitos y exigencias en los Programas, alejadas de la realidad institucional que no garantizan la pertenencia social y la pertinencia académica de los Programas.

Se halló una tendencia generalizada en afirmar que aunque se hacen Planes de Acción y de Mejoramiento en los Programas Académicos de las Universidades Públicas para alcanzar niveles de calidad educativa, existe desinterés de parte de las instituciones encargadas de diseñar las 
políticas públicas, carencia de financiamiento por parte de las organizaciones gubernamentales y poca gestión institucional para desarrollar las estrategias que permitan transformar la educación de cara a las necesidades de la sociedad.

Es preciso mencionar que los docentes catedráticos de la Facultad de Educación manifestaron que no tienen conocimiento sobre los Planes de Acción que se diseñan y desarrollan en los Programas Académicos, debido a que carecen de oportunidades para participar en estos procesos y a que no tienen espacios de interacción institucional.

La interacción de los programas de licenciatura de la Facultad de Educación con las diversas instituciones y entidades locales y nacionales es considerada por Jefes de Programa, docentes y estudiantes como una acción acertada que permite tener una mayor visibilidad académica, dar a conocer las prácticas educativas que se dan en la Universidad Surcolombiana y mantener vigentes los programas de movilidad estudiantil que ayudan a alcanzar los propósitos de formación profesional de los Programas Académicos.

Con respecto al proceso de evaluación que se realiza en la Facultad de Educación los Jefes de Programa y Docentes de las Licenciaturas consideran que garantiza el logro del propósito formativo de los futuros licenciados. Manifiestan que a través de una valoración se determinan las habilidades, destrezas y conocimientos propios que tiene cada uno de los integrantes de la comunidad estudiantil. Es preciso señalar, que los estudiantes consideran que el modelo evaluativo que implementan los docentes no garantiza la adquisición de aprendizajes significativos para su labor profesional, porque limita la producción intelectual obedeciendo a unos lineamientos impuestos por los docentes que están orientados a la consecución de una determinación cuantitativa.

Se evidenció que los docentes consideran que existen proyectos relacionados con el impacto del egresado en el medio, demostrando que actualmente se encuentran desempeñando su labor docente tanto en el sector privado como en el público. Por su parte, los Jefes de Programa resaltan que se debe avanzar en propuestas investigativas relacionadas con el impacto del egresado que consoliden los desarrollos institucionales de los Programas Académicos y a su vez la acreditación institucional.

En lo relacionado con la pertenencia social y la pertinencia académica de los programas que constituyen el portafolio de la Facultad de Educación de la Universidad Surcolombiana, se observa una preocupación sobre el ofrecimiento de algunos programas considerados históricos $\mathrm{y}$, la necesidad de su de-construcción en la perspectiva de replantear algunos de sus procesos, prácticas y costumbresformativas. Enloque siguese pretenderá dialogar con estas conclusiones y concretar una propuesta de cambio y transformación hacia el logro de los niveles de calidad y excelencia académica, investigativa y de desarrollo humano.

En el análisis de los Documentos Maestros, se constató que los programas de pregrado de la Facultad de Educación, fueron creados entre los años 1978-1992; en esta época se dieron una serie de transformaciones en la Educación Superior en Colombia que plantearon retos a las IES, en dicho marco la Facultad de Educación de la Universidad Surcolombiana avanzó en el ofrecimiento de nuevos y variados programas de pregrado.

Es pertinente señalar que en el municipio de Neiva ni en el Huila existían Instituciones de Educación Superior que profesionalizara a los docentes, la población estudiantil requería una formación en las áreas específicas. Algunos programas de la Facultad de Educación se gestaron en Institutos o Escuelas; el caso del Programa de Artes surgió del Instituto Huilense de Cultura y las Escuelas Danzas, Teatro, Visuales y Música. Asimismo, el Programa de Inglés nació en el instituto de Idiomas, sin embargo, este Programa se vinculó al Programa de Español y Literatura, con el propósito de brindar una formación sólida a los docentes de educación básica y secundaria. El Programa de Español y Literatura es el más antiguo de la Facultad de Educación. El Programa de Licenciatura en Pedagogía Infantil surgió de la 
Tecnología en Educación Preescolar que brindo la capacitación a los docentes en la atención de la primera infancia.

En lo referente a la situación legal, todos los programas académicos de la Facultad de Educación se crearon mediante norma interna aprobada por el Consejo Superior Universitario de la Universidad Surcolombiana, los programas adscritos a la Facultad cuentan con Registro Calificado otorgado por el Ministerio de Educación Nacional. Respecto, a la Acreditación de Alta Calidad, es conveniente señalar que en la actualidad tres programas cuentan con ella:

Programa de Educación Básica con Énfasis en Humanidades y Lengua Castellana, Programa de Licenciatura en Ingles ( 2 veces acreditado de alta calidad) y el Programa de Educación Artística y Cultural.

Es conveniente señalar que la misión de los siete programas académicos enfatiza en la formación en investigación, la cual garantiza el desarrollo de proyectos en cada una de las áreas específicas que intenta generar cambios en los procesos educativos. No obstante, en la actualidad los procesos de investigación de los programas académicos son incipientes toda vez que no se cuenta con un apoyo o apalancamiento financiero adecuado.

En cuanto a la visión, los programas de la Facultad de Educación están proyectados en el reconocimiento a nivel regional, nacional e internacional de la formación integral de los egresados, con capacidad de aportar a la educación inicial, básica y media elementos esenciales en la práctica pedagógica con el propósito, de crear e innovar estrategias y métodos que hacen parte en la práctica.

En la revisión de los Documentos Maestros, se evidenció que dichos programas deben formar a los docentes en cada una de las áreas, con el propósito de que el docente responda a las necesidades del contexto. Además, el docente debe desarrollar unas habilidades y capacidades para atender a las problemáticas y situaciones que se presentan en el mismo.
Lo que se puede deducir del análisis de los Documentos Maestros de todos los programas académicos de pregrado que ofrece la Facultad de Educación de la Universidad Surcolombiana es que éstos responden a todas las exigencias demandadas en las normas que regulan la educación superior en Colombia. No obstante, es conveniente señalar que en el proceso de construcción curricular se evidencian tres fases interdependientes, recíprocas y complementarias, estas son el diseño, el desarrollo y la evaluación curricular. Se puede afirmar que en lo referente al Diseño Curricular, los programas son coherentes con lo demandado en la normatividad, su ejecución y evaluación, serán referentes para el análisis de los otros resultados de esta investigación, como lo son, las observaciones de los pares académicos del CNA y de CONACES y la información suministrada por directivos, docentes y estudiantes de los diferentes programas de Licenciatura.

\section{Discusión}

\section{Propuesta de intervención}

Se plantean estrategias y acciones concretas para responder a los nuevos retos que enfrentan las unidades formadoras de docentes, en el contexto de del post-acuerdo. Resulta indiscutible adelantar un proceso de de- construcción de la Facultad de Educación que facilite y propicie una re-construcción de la misma a partir de su naturaleza académica, cultural, investigativa, social, política y cultural, lo cual significa entender que los procesos anclados en la tradición y el statu- quo deben intervenirse y en ese sentido es válido señalar la necesidad de un cambio en la trayectoria formativa del profesional de la Educación.

La de-construcción es asumida como el "proceso que permite des-estructurar o descomponer, incluso dislocar las estructuras que sostienen la arquitectura conceptual de un determinado sistema o de una secuencia histórica; también, des-sedimentar los estratos de sentido que ocultan la constitución genética de un proceso significante bajo la objetividad constituida". (Derrida: 1999). 
Un elemento a destacar consiste en señalar que la crisis por la que atraviesan las unidades formadoras de docentes, debe asumirse como una "opción de calidad" o como "una oportunidad". Significa entonces que la Facultad de Educación de la Universidad Surcolombiana debe encaminarse a la construcción de nuevos horizontes de acción como una impronta básica de re-construcción y re-significación de su proceso formativo y para ello es necesario intervenir de manera estructural, los siguientes factores:

Transformación de los criterios que orientan el proceso de admisión actual de los estudiantes

Se hace indispensable avanzar en un proceso de discusión, análisis y reflexión sistemática sobre la forma como se están seleccionando y admitiendo los estudiantes a los diferentes programas de Licenciatura. Es importante implementar un proceso de admisión que garantice que los estudiantes demuestren una actitud y aptitud básica para su formación como profesionales de la Educación, es necesario eliminar las segundas opciones (no entró a una carrera como primera opción y el puntaje le sirve para ingresar a otra como segunda opción) dado que no garantiza que el estudiante "quiera" ser licenciado. Se propone la elaboración de Pruebas Específicas por Programa, que pueden ser complementadas con los resultados de la Prueba Saber 11. Es necesario garantizar que la trayectoria formativa de los nuevos profesionales esté fundamentada en el quehacer docente y pedagógico, razón de ser, de las Unidades Formadoras de docentes.

Definir y estructurar un segmento curricular básicamente relacionado con el SABER PEDAGÓGICO, como saber esencial del profesional de la Educación

Si bien esta exigencia es de norma, no obstante se hace necesario que la Facultad de Educación de la Universidad Surcolombiana, retome de manera estructural la FORMACIÓN PEDAGÓGICAdesuslicenciados. Esconveniente desarrollar un proceso de Autoevaluación del actual Departamento de Psicopedagogía, con el propósito de determinar su devenir como la unidad académica-administrativa responsable de la formación pedagógica y didáctica de todos los estudiantes de la Facultad de Educación. Además de prestar servicios docentes en el área pedagógica y didáctica, el Departamento de Psicopedagogía, será el responsable de la investigación en todos los niveles como también de los programas y acciones de proyección social.

\section{Consolidar la cadena formativa en investigación Educativa}

La Facultad de Educación de la Universidad Surcolombiana debe desempeñar un papel protagónico en la consolidación de la CADENA Formativa en Investigación Educativa. A través del Centro de Investigación de Excelencia de la Facultad de Educación CIECE, creado por Acuerdo Superior No. 031 DE 2015, debe estructurar un sólido Plan de Acción que responda a lo regulado en el Plan de Desarrollo Institucional 2014-2025, que permita dinamizar y consolidar el proceso de formación en Investigación, que orientado por las Líneas de Investigación de la Facultad aprobadas por el Consejo de la Facultad de Educación, integre y afiance la labor investigativa de los programas ONDAS, Pequeños Científicos, Semilleros de Investigación, Jóvenes Investigadores, Grupos de Investigación, Redes de Investigación nacionales e internacionales.

Desarrollar un proceso de Modernización Curricular sustentado en la integralidad de sus principios misionales que responda a la formación disciplinaria, interdisciplinaria y transdisciplinaria

El Grupo de Investigación PACA Categoría A de Colciencias ha presentado ante las diferentes instancias académico-administrativas de la Universidad Surcolombiana, como resultado de una indagación sistemática y participativa, la propuesta de Modernización Curricular de la Universidad Surcolombiana sustentada en la integración e interdisciplinariedad (ISBN 978958-8682-39-6) en donde expone de manera clara, viable y pertinente la propuesta de estructurar los currículos a partir de Núcleos Temáticos y Problemáticos que permitan 
aportar a la integralidad de los programas de Licenciatura a partir de la formulación de cuatro Núcleos Temáticos y Problemáticos a saber: Fundamentación Básica, Desarrollo Aplicado, Formación Socio-Humanística y Formación en Investigación. La pertenencia social y la pertinencia académica deben ser las improntas sustantivas de todo el portafolio académico de la Facultad de Educación que integre la formación de pregrado y postgrado actualmente existente. Se deben debilitar las islas y las "territorios marcados" y dar paso a procesos de articulación, integridad holísticos y socialmente necesarios.

\section{Concretar la dinámica del CIECE de la Facultad de Educación}

El Centro de Investigación de Excelencia de la Facultad de Educación debe ser el faro orientador de la dinámica, cuyo objetivo central sea "Promover la calidad de la educación y el desarrollo humano, en la educación media, técnica y superior para estimular la participación y desarrollo de las nuevas generaciones de investigadores emprendedores, desarrolladores tecnológicos e innovadores".

Revivir y re-lanzar la Alianza con las TRES NORMALES SUPERIORES del Huila

Se hace indispensable re-activar la realización de programas y proyectos conjuntos con las tres Normales Superiores del Departamento Neiva, Gigante y Pitalito. Si se pretende consolidar la formación de profesionales de la Educación es ineludible articular las acciones, trabajos y programas con los niveles básicos o precedentes en la formación de Licenciados.

\section{Transformar el proceso de evaluación del rendimiento académico en una acción eminentemente cualitativa}

Los resultados obtenidos de la evaluación del rendimiento académico de los estudiantes desde la perspectiva cuantitativa (escalas, medidas, puntajes, otros), son desalentadores, en cuanto a la permanencia y graduación estudiantiles en la Universidad Surcolombiana, se debe dar paso a la incorporación e instauración de criterios
EMINENTEMENTE CUALITATIVOS para promocionar a nuestros futuros profesionales de la educación. De una evaluación sumativa y punitiva a un proceso de evaluación formativo y valorativo. Este debe ser un desafió esencial para la nueva Facultad de Educación, en un escenario de paz, equidad, reconciliación de cara al contexto del post-acuerdo.

Propiciar y concretar la creación del Instituto de Aplicación Pedagógica de la Facultad de Educación

Mediante convenio con la Secretaria de Educación de Neiva se propone la entrega de una Institución Educativa en comodatoa la Universidad Surcolombiana con una duración mínima de una década (10 años), que permita la creación del Instituto de Aplicación Pedagógica de la Facultad de Educación. Este Instituto se convertirá en un centro de experiencias y desarrollos investigativos en el campo pedagógico, didáctico y educativo, además servirá de centro de prácticas y formación delos futuros licenciados. La Facultad deEducación de la Universidad Surcolombiana debe consolidar su relación con el sector externo y esta propuesta es una manifestación fehaciente y coherente con esta responsabilidad social.

Participar del Proyecto Nacional de creación de la Escuela Dinámica para la Paz, la equidad y la Reconciliación en el marco del Post- acuerdo

En la actualidad cuatro Grupos de Investigación de la Universidad CRECER, Acción Motríz, Molufode y PACA participan del Proyecto Nacional de creación de la Escuela Dinámica para la Paz, la Equidad y la Reconciliación que busca proponer una escuela para la Paz, Equidad, la Convivencia Social en el Post Conflicto, tomando como base prácticas sociales significativas que le aportan a la paz, equidad y reconciliación.

La escuela dinámica es considerada como un escenario de construcción social y reconocimiento dela alteridad, que se dinamiza en la medida de las dinámicas mismas de lo social, en ella convergen todo tipo de prácticas sociales derivadas de los acuerdos sociales en torno a la paz, equidad y reconciliación que no son consideradas en la 
escuela convencional, además de la formación científica, ético/estética, política, comunicativa y ciudadana.

\section{Liderar la creación del Sistema Regional de Calidad de la Educación del Huila}

Es conveniente en este proceso de resignificacióny re-conceptualizacióndela Facultad de Educación de la Universidad Surcolombiana crear nuevos espacios de acción que permitan legitimar el reconocimiento social y cultural que de ella tiene la comunidad en general, por ello, se plantea la necesidad de liderar un proceso de afianzamiento y articulación con las diferentes entidades responsables de la Educación en el Departamento de un sólido sistema de calidad de la Educación y en esta perspectiva se plantea el desarrollo de acciones conjuntas que permitan trabajar por un Huila libre de analfabetismo y con unja educación seria, legitima, pertinente y de calidad.

Consideramos que la Acreditación de la Facultad no puede reducirse a la obtención de un acto administrativo, por el contrario se es consciente que la Acreditación de la Facultad de Educación debe ser una Acreditación y Reconocimiento por la sociedad y en ese sentido seguiremos trabajando para su obtención.

\section{Modalidades de formación alternativas (presencial, distancia y virtual)}

Es necesario incorporar los desarrollos virtuales a la formación de nuevos profesionales de la educación. Si existe una dimensión teleológica clara, precisa, viable y coherente, esta nueva incursión permitirá una visibilidad mayor de la Facultad y por ende, una nueva opción formativa para el egresado de la media en el Departamento del Huila esencialmente. La incorporación de la distancia y la virtualidad COMPLEMENTARÁ el proceso formativo que actualmente desarrolla la Facultad de Educación de la Universidad Surcolombiana.

\section{Referencias}

Almonacid, P., Montes, I., y Vásquez, J. (2009). Un Análisis factorial para evaluar la pertinencia de un programa académico desde la perspectiva de los Graduados: un estudio de Caso. Medellín, Colombia. Una Revista Latinoamericana de Economía Aplicada, 13 (29), 97-126. Recuperado de http://publicaciones.eafit.edu.co/index. php/ecos- economia/article/view/194

Cano Ramírez, A. (2005). Aspectos Para Una Definición De Evaluación [archivo PDF]. Disponible en: http://www2.ulpgc.es/hege/ almacen/download/38/38196/tema_5_ elementos_para_una_definicion_de_ evaluacion.pdf.

Cardoso, E.O., Cerecedo, M.T. y Trejo, C. (2009). Evaluación de la organización académicoadministrativa de tres programas de Maestría en Educación con relación a los parámetros establecidos por el CONACyT. Innovar, Bogotá, 19 (Supl. 1), 157-177. Recuperado de: http://www.scielo.org.co/ scielo.php?script=sci_arttext\&pid=S012150512009000400012\&lng=es\&tlng=es.

Cardoso, E. O., Ramos, J. R. y Cerecedo, M. T. (2011). Evaluación de la organización académico administrativa con base en la metodología de los sistemas suaves: El caso de la licenciatura en turismo. Revista Electrónica Educare, Enero-Junio, 57-78. Recuperado de: http://148.215.2.11/articulo. oa?id=194118804005

Casanova, M.A. (2005). Evaluación: Concepto, Tipología y Objetivos (pp. 67- 101) [archivo PDF]. Disponible en: http:// recursos.udgvirtual.udg. $\mathrm{mx} /$ biblioteca/ bitstream/20050101/1000/1/Lectura_4_ Evaluacion_concepto_tipologia_y_ objetivos.pdf\%20rel='nofollow'.

Contreras, K., Caballero, C., Palacio, J. \& Pérez, A. M. (2008). Factores asociados al fracaso académico en estudiantes universitarios de Barranquilla (Colombia). Psicología 
desde el Caribe, (22) 110-135. Recuperado de http://www.redalyc.org/articulo. oa?id=21311866008.

Consejo de Programa de Licenciatura en Ciencias Naturales (2009). Documento Para Renovación De Registro Calificado Licenciatura en Ciencias Naturales. Neiva (H), Colombia: Universidad Surcolombiana.

Consejo de Programa de Licenciatura en Educación Artística (s.f.). Documento Para Renovación De Registro Calificado Licenciatura en Educación Artística. Neiva (H), Colombia: Universidad Surcolombiana.

Consejo de Programa de Licenciatura en Educación Física, Recreación y Deportes (2014). Documento Para Renovación De Registro Calificado Licenciatura en Educación Física, Recreación y Deportes. Neiva $(\mathrm{H})$, Colombia: Universidad Surcolombiana.

Consejo de Programa de Licenciatura en Inglés (2014). Documento Para Renovación De Registro Calificado Licenciatura en Inglés. Neiva $(\mathrm{H})$, Colombia: Universidad Surcolombiana.

Consejo de Programa de Licenciatura en Lengua Castellana (s.f.). Documento Para Renovación De Registro Calificado Licenciatura en Lengua Castellana. Neiva $(\mathrm{H})$, Colombia: Universidad Surcolombiana.

Consejo de Programa de Licenciatura en Matemáticas (2009). Documento Para Renovación De Registro Calificado Licenciatura en Matemáticas. Neiva (H), Colombia: Universidad Surcolombiana.

Consejo de Programa de Licenciatura en Pedagogía Infantil (2010). Documento Para Renovación De Registro Calificado Licenciatura en Pedagogía Infantil. Neiva (H), Colombia: Universidad Surcolombiana.

Estévez Solano, C. (2000). Evaluación integral por procesos. Una experiencia construida desde y en el aula. Santa Fe de Bogotá. Cooperativa Editorial Magisterio.
Expósito, J., Olmedo, E. y Fernández, A. (2004). Patrones metodológicos en la investigación española sobre evaluación de programas educativos. Revista Electrónica de Investigación y Evaluación Educativa, v. 10, n. 2. Recuperado de http://www.uv.es/ RELIEVE/v10n2/RELIEVEv10n2_2.htm

Fontalvo, T.J., Vergara, J.C. y Mendoza, A. (2010). Evaluación de los sistemas de gestión de calidad en los programas de Ingeniería Industrial de Barranquilla. [Documento PDF]. Disponible en la dirección web http://www.google.com.co/l?sa=t\&rct=j\&q $=\&$ esrc $=$ s\&source $=$ web\&cd $=1 \&$ ved $=0 \mathrm{CBsQ}$ FjAAahUKEwiv7eHizq7IAhVJKB4KHW0J Ct8\&url=http $\% 3 \mathrm{~A} \% 2 \mathrm{~F} \% 2 \mathrm{Fdialnet}$.unirioja. es \%2Fdescarga\%2Farticulo\%2F3633991. pdf\&usg=AFQjCNFAPJ1s2jfT_VfZO8UNkNPFNBGZg\&sig2=I-g3QwV4Z_ V7ZsI2sO0asg

Gette-Ponce, S. (2007). Fundamentación teórica de la evaluación de los aprendizajes a nivel superior. (2a). Barranquilla: Editorial Uniautónoma.

Guía para evaluación de programas académicos en la Universidad de Puerto Rico. (2007). [Documento PDF]. Disponible en: http:// daarrp.uprrp.edu/daa/revision_bachillerato/ guias_de_evaluacion_sep t-07.pdf

López Cámara, V. (2003). Investigación Educativa Los procesos de evaluación en México para los programas de licenciatura en Odontología. Reencuentro, (38)83-90. Recuperadodehttp:// www.redalyc.org/articulo.oa?id=34003811

López Jiménez, N.E., Puentes De Velásquez, A.V., Caballero Sánchez, J., Bahamón Cerquera, P.E., Ducuara Manrique, A., Zúñiga Camacho, G.,..... Navarro Gutiérrez, D.M. (2006). Evaluación por competencias: Un reto por enfrentar y un proceso por construir. Colombia. Ed: Universidad Surcolombiana ISBN: 958-8154-86-3 v. 600 págs. 198

Malagón-Plata, L. (2009). La pertinencia curricular: un estudio en tres programas universitarios. Educación y Educadores, 12(1). Consultado 
de http://educacionyeducadores.unisabana. edu.co/index.php/eye/article/view/746

Memorias del simposiosobre acreditación universitaria. (1994). Santafé de Bogotá: ICFES, 1994

Nowalski, R. G. (2001). Evaluación y exclusión en la enseñanza universitaria. México D.F. Ed: Paidós Educador.

Pérez Abril, M. y Bustamante, G. (2001). Evaluación escolar ¿Resultados o procesos?. Investigación, reflexión y análisis crítico. Segunda edición. Santa Fe de Bogotá, Cooperativa Editorial Magisterio.

Red Maestros de Maestros. (s.f.). El Modelo de congruencia de Objetivos de Ralph Tyler. Recuperado de: http://www.rmm.cl/ index_sub.php?id_contenido=10090\&id_ seccion $=5884 \&$ id_portal $=709$.

Stufflebeam, D., \& Shinkfield, A. J. (1988). Evaluación Sistemática, Guía Teórica y Práctica. Madrid: Centro de Publicaciones del Ministerio de Educación y Ciencia; Ediciones Paidós Ibérica.

Universidad Nacional Abierta. Dirección de Investigaciones y Postgrado, El método evaluativo de scriven orientado hacia el consumidor, Capítulo 10. Stufflebeam, D. y Schinkfield, A. (1985). Evaluación Sistemática. Guía teórica y práctica. Colección Temas de Educación. Barcelona: Paidos.

Villavicencio, J. (2009). Evaluación del desempeño docente: la experiencia del Programa Académico de odontología de la Universidad del Valle. Colombia Médica, 39 (Supl 1 1), 57-63. Recuperado de http://colombiamedica. univalle.edu.co/index.php/comedica/ article/view/565/932

GRUPODEINVESTIGACIÓN PACA. Evaluación por competencias: Un reto por enfrentar y un proceso por construir. Facultad de Educación. Universidad Surcolombiana. Neiva, Junio de 2006.

Estévez Solano, Cayetano. Evaluación Integral por Procesos. Una experiencia construida desde y en el aula. Colección MESA REDONDA, No 41. Editorial Magisterio. Bogotá, 1998.

Pérez, Abril, M. y Bustamante. Guillermo. Evaluación Escolar ¿resultados o procesos? Investigación, Reflexión y Análisis Crítico. Colección MESA REDONDA, No. 39. Editorial Magisterio. Bogotá, 1996.

ICFES. "Simposio sobre Acreditación Universitaria". Memorias". Bogotá. 1994.

Restrepo, B. Raquel, Sánchez, P. Mauricio y Uribe, T. Alicia. Aseguramiento de la calidad en los programas de posgrado. Guía para la autoevaluación y la evaluación de pares externos. UNIVERSIDAD DE ANTIOQUIA. Segunda Edición. 2006.

Villada Osorio, Diego. Evaluación Integral de los procesos educativos. Manizales. 1997.

Bustos Cobos, Félix. La evaluación de los alumnos y el PEI. Serie CONSTRUYENDO TU PEI. Lineamientos para las comunidades Educativas. Bogotá. 1996

Roa Varelo, A. y Pacheco, Iván. EDUCACION SUPERIOR EN COLOMBIA: doce propuestas para la próxima década. Boston College. Center International de High Education, Universidad del Norte. Barranquilla. 2014

GRUPO DE INVESTIGACION PACA. Evaluación de la Calidad de la Educación en Colombia. Estado del Arte. Volumen I y II. Universidad Surcolombiana. Universidad Surcolombiana. Neiva. 2007 y 2010. 